\title{
Synthesis of new optically pure isoxazolines via 1,3-dipolar cycloaddition of nitrile oxides with allyl esters derived from eugenol
}

\author{
Ghada Lahouar, Amira Bahy, Ridha Touati and Bechir Ben Hasine *
}

Laboratoire de Synthèse Organique Asymétrique et Catalyse Homogène (11URES56), Faculté des Sciences de Monastir, Université de Monastir, Bd., de l'Environnement, 5019 Monastir, Tunisia

${ }^{*}$ Correspondence to B. Ben Hasssine Tel.: +216 73500 279; Fax: +216 73500 278. E-mail address: bechirbenhassine@yahoo.fr bechirbenhassine@yahoo.fr

\begin{abstract}
1,3-dipolar cycloaddition of arylnitrile oxides with allyl ester prepared from eugenol afforded new chiral isoxazolines in good yields. The chemical structure of this compounds was characterized by ${ }^{1} \mathrm{H} N M R,{ }^{13} \mathrm{C}$ NMR, $2 \mathrm{D}$ NMR and TOF-MS analysis. All the cycloadducts were obtained through a regiospecific and stereospecific pathway and all cases, only one isomer was isolated, as established by unambiguous NMR analysis.
\end{abstract}

\section{Keywords}

1,3-dipolar cycloaddition, arylnitrile oxides, allyl esters, isoxazolines, regiospecific reaction.

\section{Academic Discipline And Sub-Disciplines}

Chemistry

\section{SUBJECT CLASSIFICATION}

Organic Synthesis

\section{TYPE (METHOD/APPROACH)}

\section{1,3-dipolar cycloaddition reactions}

\section{INTRODUCTION}

Heterocyclic compounds have a wide range of applications in synthetic organic chemistry. Especially, isoxazolines are of considerable interest due to their versatile applications in pharmaceutical and agrochemical fields. Isoxazoline derivatives have been reported to possess anti influenza virus,[1] glycoprotein Ilb/lla receptor antagonists,[2] antidiabetic,[3] antitumour,[4] antifungal,[5] analgesic, anti-inflammatory,[6] spermicidal and anti HIV,[7] $\beta$-adrenergic receptor antagonist,[8] antistress[9] and anticancer properties.[10]

In fact, the combrestatine A-4, analogous 1-3 and the Avicine 4 compound derived from isoxazolines possess anti-cancer activities.[11]

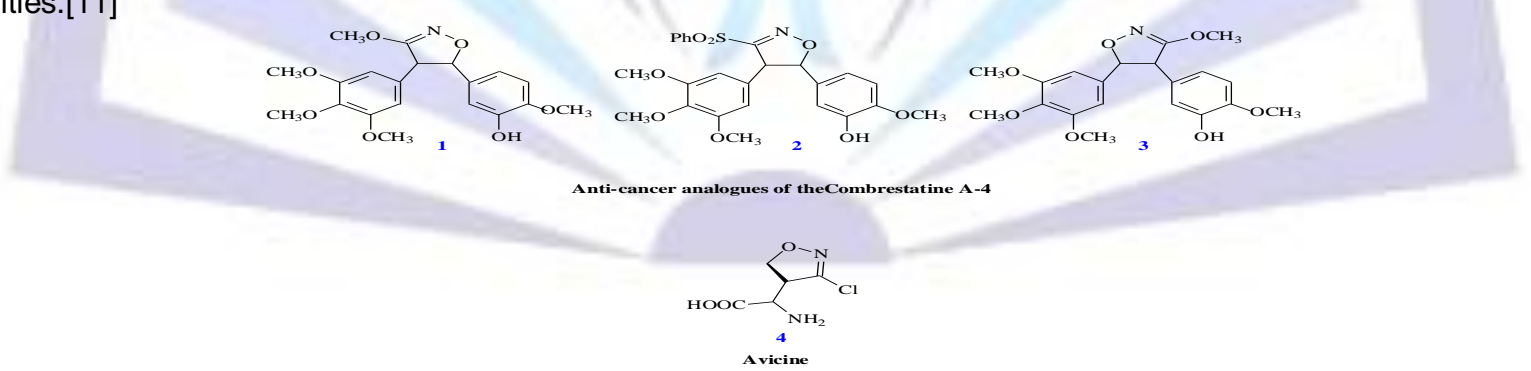

Figure 1- Representative examples of anti-cancer isoxazoline derivatives.

The 1,3-dipolar cycloaddition reactions represent one of the best method for the preparation of five-membered heterocycles,[12] and natural products.[13] In particular the 1,3-dipolar cycloaddition of nitrile oxides with alkenes and alkynes afforded isoxazolines which are used as intermediates for the synthesis of $\beta$-amino alcohols and alkaloids.[14,15] In order to increase the activities of the cycloadducts, we have used the eugenol [4-allyl-2methoxyphenol] as a precursseur of the dipolarophiles because it is well known that this natural compound have different biological activities such as antispasmodic,[16] antipyretic,[17] anti-inflammatory,[18] and antibacterial activities.[19]

Herein, we report an efficient and practical procedure for the preparation of new optically pure isoxazolines 7(ad-cf) via the 1,3-dipolar cycloaddition of the arylnitrile oxides with esters, synthesized from eugenol and having a terminal double bond. The reaction was carried out in toluene at $80^{\circ} \mathrm{C}$ without a catalyst.

\section{Results and discussion}

Dipolarophiles 5(a-c) were prepared in two steps (Scheme1). The first one concern the synthesis of the $N$-protected aminoacids. The condensation of the later compounds with eugenol gave the corresponding esters.[20] 
Journal of Advances in Chemistry
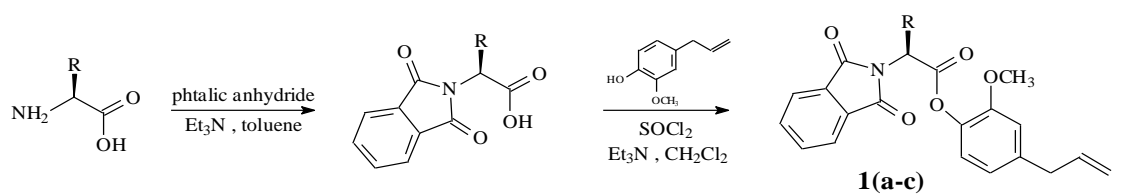

Scheme 1- Synthesis of the optically pure esters derived from eugenol and amino acid.

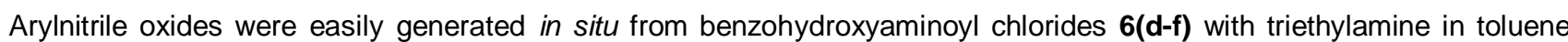
according to a known procedure.[21] The cycloaddition reaction of dipolarophiles $5(a-c)$ with the arylnitrile oxides at reflux of toluene for 48h (Scheme2) afforded the isoxazolines 7(ad-cf) with good yields as indicated in table 1.

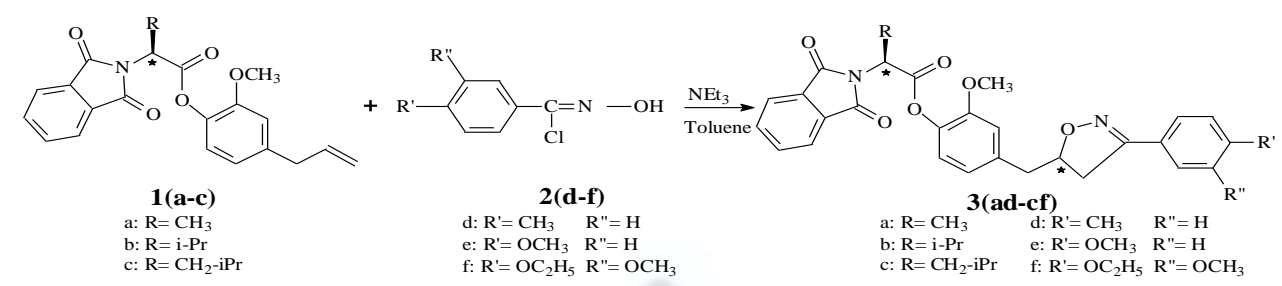

Scheme 2-1,3-Dipolar cycloaddition reaction of benzohydroxyaminoyl chlorides 6(d-f) with allyl esters 5(a-c).

Table 1. Yields of cycloadducts $\mathbf{3}(\mathrm{ad}-\mathrm{cf})$

\begin{tabular}{|c|c|c|c|c|c|}
\hline Entry & $\mathbf{R}$ & $\mathbf{R}^{\prime}$ & $\mathbf{R}$ & Cycloadducts & Yields \% \\
\hline $\mathbf{1}$ & $\mathrm{CH}_{3}$ & $\mathrm{CH}_{3}$ & $\mathrm{H}$ & $\mathbf{3 a d}$ & $58 \%$ \\
\hline $\mathbf{2}$ & $\mathrm{CH}_{3}$ & $\mathrm{OCH}_{3}$ & $\mathrm{H}$ & $\mathbf{3 a e}$ & $72 \%$ \\
\hline $\mathbf{3}$ & $\mathrm{CH}_{3}$ & $\mathrm{OC}_{2} \mathrm{H}_{5}$ & $\mathrm{OCH}_{3}$ & $\mathbf{3 a f}$ & $61 \%$ \\
\hline $\mathbf{4}$ & $\mathrm{i}-\mathrm{Pr}$ & $\mathrm{CH}_{3}$ & $\mathrm{H}$ & $\mathbf{3 b d}$ & $62 \%$ \\
\hline $\mathbf{5}$ & $\mathrm{i}-\mathrm{Pr}$ & $\mathrm{OCH}_{3}$ & $\mathrm{H}$ & $\mathbf{3 b e}$ & $60 \%$ \\
\hline $\mathbf{6}$ & $\mathrm{i}-\mathrm{Pr}$ & $\mathrm{OC}_{2} \mathrm{H}_{5}$ & $\mathrm{OCH}_{3}$ & $\mathbf{3 b f}$ & $65 \%$ \\
\hline $\mathbf{7}$ & $\mathrm{CH}_{2}-\mathrm{i}-\mathrm{Pr}$ & $\mathrm{CH}_{3}$ & $\mathrm{H}$ & $\mathbf{3 c d}$ & $60 \%$ \\
\hline $\mathbf{8}$ & $\mathrm{CH}_{2}-\mathrm{i}-\mathrm{Pr}$ & $\mathrm{OCH}_{3}$ & $\mathrm{H}$ & $\mathbf{3 c e}$ & $68 \%$ \\
\hline $\mathbf{9}$ & $\mathrm{CH}_{2}-\mathrm{i}-\mathrm{Pr}$ & $\mathrm{OC}_{2} \mathrm{H}_{5}$ & $\mathrm{OCH}_{3}$ & $\mathbf{3 c f}$ & $62 \%$ \\
\hline
\end{tabular}

\section{Regiochemistry and stereochemistry of the cycloaddition}

Two possible regioisomers I and II can be theoretically formed (Scheme 3) [22]. In practice, we have obtained only one product as evidenced by TLC and ${ }^{1} \mathrm{H}$ NMR examination of the crude mixture. Cycloadducts 7 (ad-cf) have been purified by a column chromatography and characterized by NMR $\left({ }^{1} \mathrm{H}\right.$ and $\left.{ }^{13} \mathrm{C}\right)$. Based on the literature $[23]$ and the ${ }^{13} \mathrm{C}$ NMR, we can conclude that the new cycloadducts possess the same stereochemistry as detailed below. The cycloaddition of the amino esters 7(ad-cf) with the arylnitrile oxides led to new cycloadducts having a new chiral center: the quaternary carbon linked to the substituted phenyl group of the isoxazoline ring.

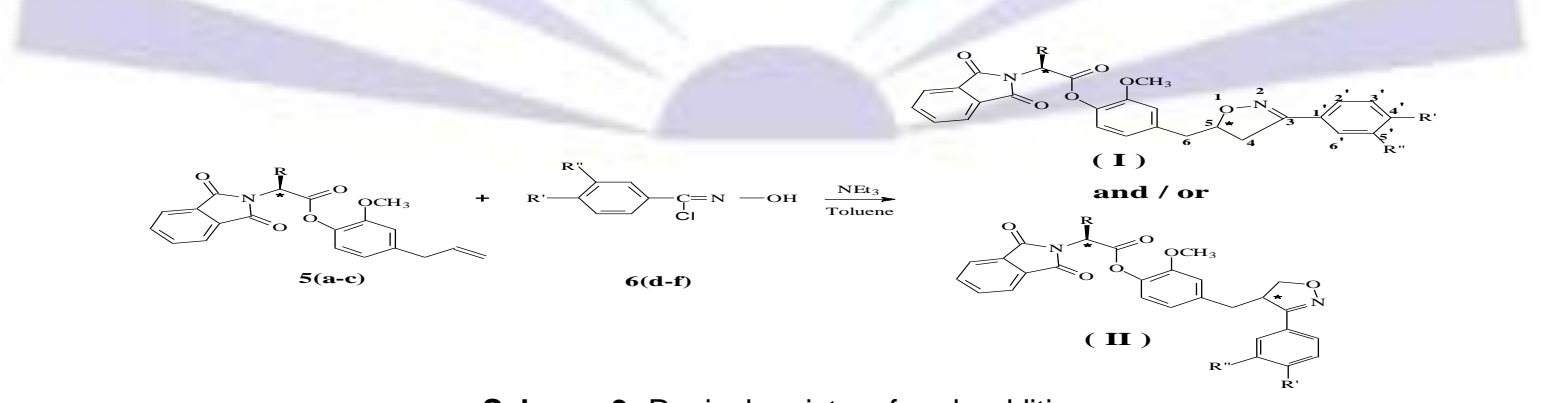

Scheme 3- Regiochemistry of cycloaddition.

Compound 7cd was obtained as a yellow liquid. Its positive ES-MS showed a pseudo-molecular ion peak $[\mathrm{M}+\mathrm{H}]^{+}$at $\mathrm{m} / \mathrm{z}=$ 541 compatible with the molecular formula $\mathrm{C}_{32} \mathrm{H}_{32} \mathrm{~N}_{2} \mathrm{O}_{6}$. The structure was confirmed by the disappearance in the ${ }^{1} \mathrm{HNMR}$ spectrum of the signal at $\delta_{\mathrm{H}} 5.01 \mathrm{ppm}$ relative to the terminal ethylenic protons and the appearance of a doublet of doubets signal at $\delta_{\mathrm{H}} 2.98\left(\mathrm{dd}, 1 \mathrm{H}^{\prime}, J_{1}=7.8 \mathrm{~Hz}, J_{2}=16.5 \mathrm{~Hz}\right)$ and $3.25\left(\mathrm{dd}, 1 \mathrm{H}_{4^{\prime \prime}}, J_{1}=10.2 \mathrm{~Hz}, J_{2}=16.8 \mathrm{~Hz}\right.$ ) attribuable to the proton $\mathrm{H}_{4}$ and the presence of a singlet at $\delta_{\mathrm{H}} 2.33(\mathrm{~s}, 3 \mathrm{H})$ corresponding to the methyl protons. A characteristic AA'BB' pattern for aromatic hydrogens was observed in the ${ }^{1} \mathrm{H}$ NMR spectrum. Examination at $300 \mathrm{MHz}$ offered an excellent resolution with a doublet at $\delta_{\mathrm{H}} 7.14\left(\mathrm{~d}, 2 \mathrm{H}, \mathrm{J}=8.1 \mathrm{~Hz}, \mathrm{H}_{\left.3^{\prime}, 5^{\prime}\right)}\right.$ and a second doublet at $\delta_{\mathrm{H}} 7.48\left(\mathrm{~d}, 2 \mathrm{H}, \mathrm{J}=8.1 \mathrm{~Hz}, \mathrm{H}_{2^{\prime}, 6^{\prime}}\right)$. The ${ }^{13} \mathrm{C}$ NMR spectrum confirmed the above spectral data by the observation of signals at $126.0\left(\mathrm{C}_{1^{\prime}}\right), 126.2\left(\mathrm{C}_{2^{\prime}, 6^{\prime}}\right)$, $128.8\left(\mathrm{C}_{3^{\prime}, 5^{\prime}}\right)$ and $139.7\left(\mathrm{C}_{4}\right)$ ppm relative to the carbons of the $p$-substituted aromatic system. The same spectrum 
Journal of Advances in Chemistry

showed signals at $\delta_{c} 80.9$ and 155.9 atributable to the bearing oxygen carbon $\mathrm{C}_{5}$ and $\mathrm{C}_{3}$ of imine fonction, respectively. In addition, a whole set of linkages confirming the molecular structure of compound 7cd was reinforced by the HMB spectrum which showed correlations between the proton $\mathrm{H}_{4}$ and $\mathrm{C}_{1^{\prime}}, \mathrm{C}_{3}, \mathrm{C}_{5}$ and $\mathrm{C}_{6}$ as well as correlations between $\mathrm{H}_{2}, 6$ and $\mathrm{C}_{1}, \mathrm{C}_{3}$ and $\mathrm{C}_{4}$. Moreover the regiochemistry was confirmed by the NOE observed between the protons $\mathrm{H}_{4}$ and the aromatic protons $\mathrm{H}_{2}, 6^{\prime}$. Although in this study the novel isoxazoline derivatives $\mathbf{7}(\mathbf{a d}-\mathrm{cf})$ were formed as an unique products indicating the regiospecificity of the reaction. Indeed the non-formation of the other 1,5-regioisomer may be explained by a possible steric crowding and by electronic factors.

\section{Experimental section}

Solvents were purified by standard methods. Melting points were determined on a Buchi SMP-20 capilary apparatus and are uncorrected. TLC was carried out on a Merck $60 \mathrm{~F}-254$ precoated silica gel plates $(0.25 \mathrm{~mm})$ and column chromatography was performed with Merck silica gel (70-230 mesh). NMR spectra were recorded on a Bruker AC-300 spectrometer ( ${ }^{\mathrm{H}} \mathrm{NMR}$ at $300 \mathrm{MHz}$ and ${ }^{13} \mathrm{C} \mathrm{NMR}$ at $75.5 \mathrm{MHz}$ ) with $\mathrm{CDCl}_{3}$ as solvent and TMS as internal standard reference. $\mathrm{Et}_{3} \mathrm{~N}$ was purchased from Acros. All starting protected amino esters were prepared according to the procedure.[21] In all cases, the crude amino esters was purified before use.[24] The benzohydroxyaminoyl chlorides 6(d-f) were prepared according to the literature procedures.[22]

\section{General procedure for the preparation of the new isoxazolines}

A magnetically stirred solution of amino esters (5a-c) and the appropriate precursor of the benzohydroxyaminoyl chlorides in dry toluene, was refluxed under nitrogen for $15 \mathrm{~min}$. $\mathrm{Et}_{3} \mathrm{~N}(2 \mathrm{~mL})$ was then added and the mixture was stirred and refluxed for $48 \mathrm{~h}$. After the filtration of the triethylamine hydrochloride, the solvent was evaporated and the residue was purified by silica gel column chromatography (eluent: cyclohexane-AcOEt, 70:30).[25]

2-methoxy-4-\{[3-(4-methoxyphenyl)-4,5-dihydroisoxazol-5-yl]methyl\}phenyl2-(1,3-dioxo-1,3-dihydro-2H-isoindol-2yl)propanoate(7ae)

Yellow liquid (72\%). ${ }^{1} \boldsymbol{H}$ NMR $\left(300 \mathrm{MHz}, \mathrm{CDCl}_{3}\right): \delta=1.72(\mathrm{~d}, 3 \mathrm{H},-\mathrm{CH} 3, J=7.2 \mathrm{~Hz}), 2.81-2.88\left(\mathrm{dd}, 1 \mathrm{H}, J_{1}=6.9 \mathrm{~Hz}, J_{2}=14.1\right.$ $\mathrm{Hz}), 3.00-3.36(\mathrm{~m}, 3 \mathrm{H}), 3.76\left(\mathrm{~s}, 3 \mathrm{H},-\mathrm{OCH}_{3}\right), 3.79\left(\mathrm{~s}, 3 \mathrm{H},-\mathrm{OCH}_{3}\right), 4.95-4.99(\mathrm{~m}, 2 \mathrm{H}), 6.74(\mathrm{~d}, 1 \mathrm{H}$, aromatic, $\mathrm{J}=7.4 \mathrm{~Hz})$, $6.87(\mathrm{~d}, 1 \mathrm{H}$,aromatic, $J=7.8 \mathrm{~Hz}), 7.19-7.22(\mathrm{~m}, 2 \mathrm{H}$, aromatics $), 7.30(\mathrm{~s}, 1 \mathrm{H}$, aromatic), 7.53-7.56(m, 2H, aromatics), 7.74$7.76\left(\mathrm{~m}, 2 \mathrm{H}\right.$, aromatics), 7.87-7.90(m, 2H, aromatics),. ${ }^{13} \mathrm{C} \mathrm{NMR}\left(75.5 \mathrm{MHz}, \mathrm{CDCl}_{3}\right): \delta=20.9\left(\mathrm{CH}_{3}\right), 38.9\left(\mathrm{CH}_{2}\right), 40.1\left(\mathrm{CH}_{2}\right)$, $50.8(\mathrm{OOC}-\mathrm{CH}-\mathrm{N}), \quad 55.3\left(\mathrm{OCH}_{3}\right), \quad 55.4\left(\mathrm{OCH}_{3}\right), \quad 81.3(\mathrm{CH}-\mathrm{O}), \quad 111.4\left(\mathrm{CH}_{\text {aromatic }}\right), \quad 113.8\left(2 \mathrm{CH}_{\text {aromatic }}\right), \quad 121.5\left(\mathrm{CH}_{\text {aromatic }}\right)$, 123.0 $\left(\mathrm{CH}_{\text {aromatic }}\right), 126.0\left(\mathrm{C}_{\text {aromatic }}\right), 127.6\left(\mathrm{C}_{\text {aromatic }}\right), 128.6\left(2 \mathrm{CH}_{\text {aromatic }}\right), 129.0\left(2 \mathrm{CH}_{\text {aromatic }}\right), 131.4\left(\mathrm{C}_{\text {aromatic }}\right), 133.8\left(2 \mathrm{CH}_{\text {aromatic }}\right)$, 137.7(C (Caromatic), 139.9(C (Camatic), 151.2(C $\left.\mathrm{C}_{\text {aromatic }}\right), 156.0(\mathrm{C}=\mathrm{N}), 167.4(\mathrm{~N}-\mathrm{C}=\mathrm{O}), 170.8(\mathrm{COO})$. TOFMS ES ${ }^{+}$for $\mathrm{C}_{29} \mathrm{H}_{26} \mathrm{~N}_{2} \mathrm{O}_{7}$ theoretical $[\mathrm{M}+\mathrm{H}]^{+}: 515.1740 ;$ measured $[\mathrm{M}+\mathrm{H}]^{+}: 514.1743$

2-methoxy-4-\{[3-(4-methoxyphenyl)-4,5-dihydroisoxazol-5-yl]methyl\}phenyl2-(1,3 dioxo-1,3-dihydro-2H-isoindol-2yl)-3-methylbutanoate (7be)

Yellow liquid $(60 \%) .{ }^{1} \boldsymbol{H}$ NMR $\left(300 \mathrm{MHz}, \mathrm{CDCl}_{3}\right): \delta=1.01(\mathrm{~d}, 3 \mathrm{H},-\mathrm{CH} 3, \mathrm{~J}=6.6 \mathrm{~Hz}), 1.25(\mathrm{~d}, 3 \mathrm{H},-\mathrm{CH} 3, \mathrm{~J}=6.6 \mathrm{~Hz}), 2.33-$ $2.38(\mathrm{~m}, 2 \mathrm{H}), 2.97-3.29(\mathrm{~m}, 4 \mathrm{H}), 3.73\left(\mathrm{~s}, 3 \mathrm{H},-\mathrm{OCH}_{3}\right), 3.75\left(\mathrm{~s}, 3 \mathrm{H},-\mathrm{OCH}_{3}\right), 4.87-4.90(\mathrm{~m}, 1 \mathrm{H}), 6.81-6.86(\mathrm{~m}, 2 \mathrm{H}$, aromatics $)$, 6.99-7.02(dd, $1 \mathrm{H}$,aromatic, $\left.J_{1}=1.5 \mathrm{~Hz}, J_{2}=8.1 \mathrm{~Hz}\right), 7.19-7.21(\mathrm{~m}, 2 \mathrm{H}$, aromatics), 7.52-7.55(m, 2H, aromatics), 7.75$7.58\left(\mathrm{~m}, 2 \mathrm{H}\right.$, aromatics), 7.91-7.94(m, 2H, aromatics). ${ }^{13} \mathrm{C} \mathrm{NMR}\left(75 \mathrm{MHz}, \mathrm{CDCl}_{3}\right): \delta=20.3\left(\mathrm{CH}_{3}\right), 20.9\left(\mathrm{CH}_{3}\right), 28.0(\mathrm{CH})$, $38.9\left(\mathrm{CH}_{2}\right), 40.1\left(\mathrm{CH}_{2}\right), 50.1(\mathrm{OOC}-\mathrm{CH}-\mathrm{N}), 55.2\left(\mathrm{OCH}_{3}\right), 55.4\left(\mathrm{OCH}_{3}\right), 81.0(\mathrm{CH}-\mathrm{O}), 111.4\left(\mathrm{CH}_{\text {aromatic }}\right), 113.0\left(2 \mathrm{CH}_{\text {aromatic }}\right)$, 122.2 $\left(\mathrm{CH}_{\text {aromatic }}\right), 123.0\left(\mathrm{CH}_{\text {aromatic }}\right), 126.0\left(\mathrm{C}_{\text {aromatic }}\right), 128.6\left(\mathrm{C}_{\text {aromatic }}\right), 129.0\left(2 \mathrm{CH}_{\text {aromatic }}\right), 129.3\left(2 \mathrm{CH}_{\text {aromatic }}\right), 131.3\left(\mathrm{C}_{\text {aromatic }}\right)$, 133.7 $\left(2 \mathrm{CH}_{\text {aromatic }}\right), 137.8$ ( $\left.\mathrm{C}_{\text {aromatic }}\right), 139.8\left(\mathrm{C}_{\text {aromatic }}\right), 150.4\left(\mathrm{C}_{\text {aromatic }}\right), 156.0(\mathrm{C}=\mathrm{N}), 167.2(\mathrm{~N}-\mathrm{C}=\mathrm{O}), 171.4(\mathrm{COO})$. TOFMS ES ${ }^{+}$ for $\mathrm{C}_{31} \mathrm{H}_{30} \mathrm{~N}_{2} \mathrm{O}_{7}$ theoretical $[\mathrm{M}+\mathrm{H}]^{+}: 525.2053$; measured $[\mathrm{M}+\mathrm{H}]^{+}: 525.2057$

2-methoxy-4-\{[3-(4-methoxyphenyl)-4,5-dihydroisoxazol-5-yl]methyl\}phenyl 2-(1,3-dioxo-1,3-dihydro-2H-isoindol2-yl)-4-methylpentanoate (7ce)

Yellow liquid (68\%). ${ }^{1} \boldsymbol{H}$ NMR $\left(300 \mathrm{MHz}, \mathrm{CDCl}_{3}\right): \delta=0.99(\mathrm{~d}, 3 \mathrm{H},-\mathrm{CH} 3, J=6.6 \mathrm{~Hz}), 1.03(\mathrm{~d}, 3 \mathrm{H},-\mathrm{CH} 3, \mathrm{~J}=6.6 \mathrm{~Hz}), 1.59$ $1.62(\mathrm{~m}, 1 \mathrm{H}), 2.06-2.17(\mathrm{~m}, 2 \mathrm{H}), 2.97-3.36(\mathrm{~m}, 4 \mathrm{H}), 3.79\left(\mathrm{~s}, 3 \mathrm{H},-\mathrm{OCH}_{3}\right), 3.89\left(\mathrm{~s}, 3 \mathrm{H},-\mathrm{OCH}_{3}\right), 4.93-4.98(\mathrm{~m}, 1 \mathrm{H}), 5.23-5.29(\mathrm{dd}$, $\left.1 \mathrm{H}, J_{1}=4.2 \mathrm{~Hz}, J_{2}=11.4 \mathrm{~Hz}\right), 6.72-6.88\left(\mathrm{~m}, 2 \mathrm{H}\right.$, aromatics), 6.99-7.02(dd, $1 \mathrm{H}$, aromatic, $\left.J_{1}=1.5 \mathrm{~Hz}, J_{2}=8.1 \mathrm{~Hz}\right), 7.19(\mathrm{~s}$, $1 \mathrm{H}), 7.74-7.78\left(\mathrm{~m}, 3 \mathrm{H}\right.$, aromatics), 7.86-7.92(m, $2 \mathrm{H}$, aromatics), 7.97-8.00(m, 2H, aromatics). ${ }^{13} \mathrm{C} \mathrm{NMR}(75.5 \mathrm{MHz}, \mathrm{CDCl})$ : $\delta=20.9\left(\mathrm{CH}_{3}\right), 21.2\left(\mathrm{CH}_{3}\right), 22.7(\mathrm{CH}), 38.9\left(\mathrm{CH}_{2}\right), 39.0\left(\mathrm{CH}_{2}\right), 40.3\left(\mathrm{CH}_{2}\right), 50.0(\mathrm{OOC}-\mathrm{CH}-\mathrm{N}), 55.4\left(\mathrm{OCH}_{3}\right), 55.6\left(\mathrm{OCH}_{3}\right)$, 81.0( $\mathrm{CH}-\mathrm{O}), 111.4\left(\mathrm{CH}_{\text {aromatic }}\right), 113.8\left(2 \mathrm{CH}_{\text {aromatic }}\right), 121.5\left(\mathrm{CH}_{\text {aromatic }}\right), 123.0\left(\mathrm{CH}_{\text {aromatic }}\right), 126.0\left(\mathrm{C}_{\text {aromatic }}\right), 126.2\left(\mathrm{C}_{\text {aromatic }}\right)$, $128.9\left(2 \mathrm{CH}_{\text {aromatic }}\right), 129.0\left(2 \mathrm{CH}_{\text {aromatic }}\right), 129.7\left(\mathrm{C}_{\text {aromatic }}\right), 133.7\left(2 \mathrm{CH}_{\text {aromatic }}\right), 137.8\left(\mathrm{C}_{\text {aromatic }}\right), 139.8\left(\mathrm{C}_{\text {aromatic }}\right), 150.4\left(\mathrm{C}_{\text {aromatic }}\right)$, 156.0 $(\mathrm{C}=\mathrm{N}), 167.2(\mathrm{~N}-\mathrm{C}=\mathrm{O}), 170.8(\mathrm{COO})$. TOFMS ES ${ }^{+}$for $\mathrm{C}_{32} \mathrm{H}_{32} \mathrm{~N}_{2} \mathrm{O}_{7}$ theoretical $[\mathrm{M}+\mathrm{H}]^{+}: 557.2209 ;$ measured $[\mathrm{M}+\mathrm{H}]^{+}$: 557.2205 .

2-methoxy-4-\{[3-(4-methylphenyl)-4,5-dihydroisoxazol-5-yl]methyl\}phenyl 2-(1,3-dioxo-1,3-dihydro-2H-isoindol-2yl)propanoate (7ad)

Yellow liquid (58\%). ${ }^{1} \boldsymbol{H}$ NMR $\left(300 \mathrm{MHz}, \mathrm{CDCl}_{3}\right): \delta=1.86\left(\mathrm{~d}, 3 \mathrm{H},-\mathrm{CH}_{3}, J=7.2 \mathrm{~Hz}\right), 2.39\left(\mathrm{~s}, 3 \mathrm{H},-\mathrm{CH}_{3}\right), 2.84-2.91\left(\mathrm{dd}, 1 \mathrm{H}, J_{1}=\right.$ $6.9 \mathrm{~Hz}, J_{2}=14.1 \mathrm{~Hz}$ ), 2.99-3.07(dd, $\left.1 \mathrm{H}, J_{1}=7.8 \mathrm{~Hz}, J_{2}=16.5 \mathrm{~Hz}\right), 3.12-3.18\left(\mathrm{dd}, 1 \mathrm{H}, J_{1}=6.3 \mathrm{~Hz}, J_{2}=13.8 \mathrm{~Hz}\right), 3.28-3.36(\mathrm{dd}$, $\left.1 \mathrm{H}, J_{1}=8.1 \mathrm{~Hz}, J_{2}=17.2 \mathrm{~Hz}\right), 3.82\left(\mathrm{~s}, 3 \mathrm{H},-\mathrm{OCH}_{3}\right), 4.93-4.99(\mathrm{~m}, 1 \mathrm{H}), 5.30(\mathrm{q}, 1 \mathrm{H}, J=9.9 \mathrm{~Hz}), 6.81-6.84\left(\mathrm{dd}, 1 \mathrm{H}, J_{1}=1.5 \mathrm{~Hz}\right.$, $\left.J_{2}=8.1 \mathrm{~Hz}\right), 6.89\left(\mathrm{~s}, 1 \mathrm{H}\right.$, aromatic), 7.02-7.05(dd, $\left.1 \mathrm{H}, J_{1}=1.5 \mathrm{~Hz}, J_{2}=8.1 \mathrm{~Hz}\right), 7.21(\mathrm{~d}, 2 \mathrm{H}$, aromatics, $J=8.1 \mathrm{~Hz}), 7.55(\mathrm{~d}, 2 \mathrm{H}$, aromatics, $J=8.1 \mathrm{~Hz}), 7.75-7.79(\mathrm{~m}, 2 \mathrm{H}$, aromatics $), 7.89-7.94(\mathrm{~m}, 2 \mathrm{H}$, aromatics $) .{ }^{13} \boldsymbol{C} \mathbf{N M R}\left(75.5 \mathrm{MHz}, \mathrm{CDCl}_{3}\right): \delta=$

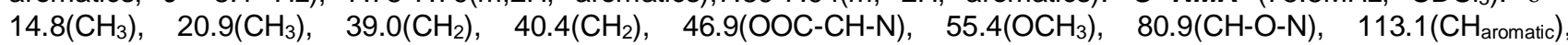
113.1 $\left(\mathrm{CH}_{\text {aromatic }}\right), 119.5\left(\mathrm{CH}_{\text {aromatic }}\right), 123.0\left(2 \mathrm{CH}_{\text {aromatic }}\right), 126.0\left(\mathrm{C}_{\text {aromatic }}\right), 126.2\left(\mathrm{CH}_{\text {aromatic }}\right), 128.8\left(2 \mathrm{CH}_{\text {aromatic }}\right), 128.8\left(\mathrm{C}_{\text {aromatic }}\right)$, $131.5\left(\mathrm{CH}_{\text {aromatic }}\right), 133.6\left(\mathrm{C}_{\text {aromatic }}\right), 135.7\left(\mathrm{C}_{\text {aromatic }}\right), 136.1\left(\mathrm{C}_{\text {aromatic }}\right), 150.4\left(\mathrm{C}_{\text {aromatic }}\right), 155.9(\mathrm{C}=\mathrm{O}), 166.8(\mathrm{~N}-\mathrm{C}=\mathrm{O}), 167.5(\mathrm{COO})$. TOFMS ES ${ }^{+}$for $\mathrm{C}_{29} \mathrm{H}_{26} \mathrm{~N}_{2} \mathrm{O}_{6}$ theoretical $[\mathrm{M}+\mathrm{H}]^{+}: 499.1790 ;$ measured $[\mathrm{M}+\mathrm{H}]^{+}: 499.1788$.

2-methoxy-4-\{[3-(4-methylphenyl)-4,5-dihydroisoxazol-5-yl]methyl\}phenyl 2-(1,3-dioxo-1,3-dihydro-2H-isoindol-2yl)-3-methylbutanoate (7bd) 
Yellow liquid (62\%). ${ }^{1} \boldsymbol{H} \mathbf{N M R}\left(300 \mathrm{MHz}, \mathrm{CDCl}_{3}\right): \delta=0.95\left(\mathrm{~d}, 3 \mathrm{H}_{\mathrm{e}},-\mathrm{CH}_{3}, \mathrm{~J}=7.3 \mathrm{~Hz}\right), 1.14-1.17(\mathrm{~m}, 4 \mathrm{H}), 2.27\left(\mathrm{~s}, 3 \mathrm{H},-\mathrm{CH}_{3}\right), 2.76-$ $3.29(\mathrm{~m}, 4 \mathrm{H}), 3.63\left(\mathrm{~s}, 3 \mathrm{H},-\mathrm{OCH}_{3}\right), 4.76-4.79(\mathrm{~m}, 2 \mathrm{H}), 6.68-6.76\left(\mathrm{~m}, 2 \mathrm{H}\right.$, aromatics), 6.89-6.92( dd, $1 \mathrm{H}$, aromatic, $\mathrm{J}_{1}=3.00 \mathrm{~Hz}$, $\left.J_{2}=8.1 \mathrm{~Hz}\right), 7.09(\mathrm{~d}, 2 \mathrm{H}$, aromatics, $J=8.1 \mathrm{~Hz}), 7.43(\mathrm{~d}, 2 \mathrm{H}$, aromatics, $J=8.1 \mathrm{~Hz}), 7.65-7.68(\mathrm{~m}, 2 \mathrm{H}$, aromatics $), 7.80-$ 7.82(m, 2H, aromatics). ${ }^{13} \mathrm{C} \mathrm{NMR}(75.5 \mathrm{MHz}, \mathrm{CDCl}): \delta=18.8\left(\mathrm{CH}_{3}\right), 20.2\left(\mathrm{CH}_{3}\right), 20.9\left(\mathrm{CH}_{3}\right), 28.0(\mathrm{CH}), 39.0\left(\mathrm{CH}_{2}\right), 40.3\left(\mathrm{CH}_{2}\right)$, 55.2( $\left(\mathrm{OCH}_{3}\right), 57.0(\mathrm{OOOC}-\mathrm{CH}-\mathrm{N}), 81.0(\mathrm{CH}-\mathrm{O}-\mathrm{N}), 113.0\left(\mathrm{CH}_{\text {aromatic }}\right), 119.5\left(\mathrm{CH}_{\text {aromatic }}\right), 120.8\left(\mathrm{CH}_{\text {aromatic }}\right), 122.2\left(2 \mathrm{CH}_{\text {aromatic }}\right)$, 123.0( $\left.\mathrm{C}_{\text {aromatic }}\right), 125.0\left(2 \mathrm{CH}_{\text {aromatic }}\right), 126.0\left(\mathrm{C}_{\text {aromatic }}\right), 126.2\left(2 \mathrm{CH}_{\text {aromatic }}\right), 128.8\left(\mathrm{C}_{\text {aromatic }}\right), 131.7\left(\mathrm{C}_{\text {aromatic }}\right), 133.7\left(\mathrm{C}_{\text {aromatic }}\right)$, 135.7(Caromatic), $150.4\left(\mathrm{C}_{\text {aromatic }}\right.$, $155.9(\mathrm{C}=\mathrm{N}), 166.4(\mathrm{~N}-\mathrm{C}=\mathrm{O}), 167.1(\mathrm{COO})$. TOFMS ES ${ }^{+}$for $\mathrm{C}_{31} \mathrm{H}_{30} \mathrm{~N}_{2} \mathrm{O}_{6}$ theoretical $[\mathrm{M}+\mathrm{H}]^{+}$: 527.2103; measured $[\mathrm{M}+\mathrm{H}]^{+}: 527.2105$

2-methoxy-4-\{[3-(4-methylphenyl)-4,5-dihydroisoxazol-5-yl]methyl\}phenyl 2-(1,3-dioxo-1,3-dihydro-2H-isoindol-2yl)-4-methylpentanoate (7cd)

Yellow liquid $(60 \%) .{ }^{1} \boldsymbol{H}$ NMR $\left(300 \mathrm{MHz}, \mathrm{CDCl}_{3}\right): \delta=0.95(\mathrm{~d}, 3 \mathrm{H},-\mathrm{CH} 3, J=6.6 \mathrm{~Hz}), 0.98(\mathrm{~d}, 3 \mathrm{H},-\mathrm{CH} 3, J=6.3 \mathrm{~Hz}), 1.53$ $1.59(\mathrm{~m}, 1 \mathrm{H}), 2.04-2.13(\mathrm{~m}, 1 \mathrm{H}), 2.33\left(\mathrm{~s}, 3 \mathrm{H},-\mathrm{CH}_{3}\right), 2.39-2.50(\mathrm{~m}, 1 \mathrm{H}), 2.79-2.84\left(\mathrm{dd}, 1 \mathrm{H}, J_{1}=6.6 \mathrm{~Hz}, J_{2}=13.8 \mathrm{~Hz}\right), 2.92-$ $3.00\left(\mathrm{dd}, 1 \mathrm{H}, J_{1}=7.8 \mathrm{~Hz}, J_{2}=16.5 \mathrm{~Hz}\right), 3.04-3.11\left(\mathrm{dd}, 1 \mathrm{H}, J_{1}=6.3 \mathrm{~Hz}, J_{2}=13.8 \mathrm{~Hz}\right.$ ), 3.21-3.30(dd, $1 \mathrm{H}, J_{1}=10.2 \mathrm{~Hz}, J_{2}=16.8$ $\mathrm{Hz}), 3.74\left(\mathrm{~s}, 3 \mathrm{H},-\mathrm{OCH}_{3}\right), 4.86-4.92(\mathrm{~m}, 1 \mathrm{H}), 5.18-5.23\left(\mathrm{dd}, 1 \mathrm{H}, J_{1}=4.5 \mathrm{~Hz}, J_{2}=11.1 \mathrm{~Hz}\right), 6.75(\mathrm{~d}, 1 \mathrm{H}, J=7.8 \mathrm{~Hz}), 6.82(\mathrm{~s}, 1 \mathrm{H})$, 6.94-6.97(dd, $\left.1 \mathrm{H}, J_{1}=0.9 \mathrm{~Hz}, J_{2}=7.8 \mathrm{~Hz}\right), 7.14(\mathrm{~d}, 2 \mathrm{H}$, aromatics, $J=8.1 \mathrm{~Hz}), 7.48(\mathrm{~d}, 2 \mathrm{H}$, aromatics, $J=8.1 \mathrm{~Hz}), 7.69-$ $7.72\left(\mathrm{~m}, 2 \mathrm{H}\right.$, aromatics), 7.82-7.86(m, $2 \mathrm{H}$, aromatics). ${ }^{13} \mathrm{C} \mathrm{NMR}\left(75 \mathrm{MHz}, \mathrm{CDCl}_{3}\right): \delta=20.65,20.90,22.67,24.64,36.82$, $39.08,40.38,50.12,55.44,80.99,113.12,120.80,122.09,123.01,126.07,126.29,128.88,131.41,133.67,135.78$, 137.96, 139.76, 150.45, 155.96, 167.14, 167.62. TOFMS ES ${ }^{+}$for $\mathrm{C}_{32} \mathrm{H}_{32} \mathrm{~N}_{2} \mathrm{O}_{6}$ theoretical $[\mathrm{M}+\mathrm{H}]^{+}: 541.2349$; measured $[\mathrm{M}+\mathrm{H}]^{+}: 541.2346$.

4-\{[3-(4-ethoxy-3-methoxyphenyl)-4,5-dihydroisoxazol-5-yl]methyl\}-2-methoxyphenyl 2-(1,3-dioxo-1,3-dihydro-2H isoindol-2-yl)propanoate (7af)

Yellow liquid (61\%). ${ }^{1} \boldsymbol{H}$ NMR $\left(300 \mathrm{MHz}, \mathrm{CDCl}_{3}\right): \delta=1.41\left(\mathrm{t}, 3 \mathrm{H},-\mathrm{CH}_{3}, J=7.2 \mathrm{~Hz}\right), 1.79(\mathrm{~d}, 3 \mathrm{H},-\mathrm{CH} 3, \mathrm{~J}=7.2 \mathrm{~Hz}), 2.76-$ $2.83\left(\mathrm{dd}, 1 \mathrm{H}, J_{1}=6.9 \mathrm{~Hz}, J_{2}=13.8 \mathrm{~Hz}\right), 2.90-2.98\left(\mathrm{dd}, 1 \mathrm{H}, J_{1}=8.1 \mathrm{~Hz}, J_{2}=16.8 \mathrm{~Hz}\right), 3.02-3.09\left(\mathrm{dd}, 1 \mathrm{H}, J_{1}=6.3 \mathrm{~Hz}, J_{2}=14.1\right.$ $\mathrm{Hz}), 3.19-3.28\left(\mathrm{dd}, 1 \mathrm{H}, J_{1}=9.9 \mathrm{~Hz}, \mathrm{~J}_{2}=16.5 \mathrm{~Hz}\right), 3.74\left(\mathrm{~s}, 3 \mathrm{H},-\mathrm{OCH}_{3}\right), 3.83\left(\mathrm{~s}, 3 \mathrm{H},-\mathrm{OCH}_{3}\right), 4.06\left(\mathrm{q}, 2 \mathrm{H},-\mathrm{OCH}_{2}, J=7.2 \mathrm{~Hz}\right)$, 4.84-4.90(m, $1 \mathrm{H}), 5.22(\mathrm{q}, 1 \mathrm{H}, J=7.5 \mathrm{~Hz}), 6.74-6.79(\mathrm{~m}, 2 \mathrm{H}$, aromatics), 6.83(s, $1 \mathrm{H}$, aromatic), 6.90-6.985(m, $2 \mathrm{H}$, aromatics), $7.29(\mathrm{~d}, 1 \mathrm{H}, \mathrm{J}=1.5 \mathrm{~Hz}), 7.66-7.71\left(\mathrm{~m}, 2 \mathrm{H}\right.$, aromatics), $7.81-7.84\left(\mathrm{~m}, 2 \mathrm{H}\right.$, aromatics) ${ }^{13}{ }^{13} \mathbf{N M R}\left(75.5 \mathrm{MHz}, \mathrm{CDCl}_{3}\right)$ : $\delta=14.1\left(\mathrm{CH}_{3}\right), 14.8\left(\mathrm{CH}_{3}\right), 39.0\left(\mathrm{CH}_{2}\right), 40.3\left(\mathrm{CH}_{2}\right), 46.9(\mathrm{OOC}-\mathrm{CH}-\mathrm{N}), 55.4\left(\mathrm{OCH}_{3}\right), 55.3\left(\mathrm{OCH}_{3}\right), 63.7\left(\mathrm{O}-\mathrm{CH}_{2}\right), 80.9(\mathrm{CH}-\mathrm{O})$, 108.4 $\left(\mathrm{CH}_{\text {aromatic }}\right), 111.2\left(\mathrm{CH}_{\text {aromatic }}\right), 113.1\left(\mathrm{CH}_{\text {aromatic }}\right), 119.71\left(\mathrm{CH}_{\text {aromatic }}\right), 120.8\left(\mathrm{CH}_{\text {aromatic }}\right), 121.7\left(\mathrm{C}_{\text {aromatic }}\right), 122.0\left(\mathrm{C}_{\text {aromatic }}\right)$, $122.9\left(2 \mathrm{CH}_{\text {aromatic }}\right), 131.4\left(\mathrm{CH}_{\text {aromatic }}\right), 133.6\left(2 \mathrm{CH}_{\text {aromatic }}\right), 135.8\left(\mathrm{C}_{\text {aromatic }}\right), 137.9\left(\mathrm{C}_{\text {aromatic }}\right), 148.8\left(\mathrm{C}_{\text {aromatic }}\right), 149.6\left(\mathrm{C}_{\text {aromatic }}\right)$, 150.4( $\mathrm{C}_{\text {aromatic }}$, $155.7(\mathrm{C}=\mathrm{N}), 166.7(\mathrm{~N}-\mathrm{C}=\mathrm{O}), 167.5(\mathrm{COO})$. TOFMS ES ${ }^{+}$for $\mathrm{C}_{31} \mathrm{H}_{30} \mathrm{~N}_{2} \mathrm{O}_{8}$ theoretical $[\mathrm{M}+\mathrm{H}]^{+}: 559.2002$; measured $[\mathrm{M}+\mathrm{H}]^{+}: 559.2006$.

4-\{[3-(4-ethoxy-3-methoxyphenyl)-4,5-dihydroisoxazol-5-yl]methyl\}-2-methoxyphenyl 2-(1,3-dioxo-1,3-dihydro-2H isoindol-2-yl)-3-methylbutanoate (7bf)

Yellow liquid (65\%). ${ }^{1} \boldsymbol{H}$ NMR $\left(300 \mathrm{MHz}, \mathrm{CDCl}_{3}\right): \delta=0.96(\mathrm{~d}, 3 \mathrm{H}, \mathrm{J}=6.6 \mathrm{~Hz}), 1.19\left(\mathrm{~d}, 3 \mathrm{H},-\mathrm{CH}_{3}, \mathrm{~J}=6.6 \mathrm{~Hz}\right), 1.42\left(\mathrm{t}, 3 \mathrm{H},-\mathrm{CH}_{3}\right.$, $\mathrm{J}=6.9 \mathrm{~Hz}), 2.77-3.09(\mathrm{~m}, 4 \mathrm{H}), 3.19-3.28\left(\mathrm{dd}, 1 \mathrm{H}, \mathrm{J}_{1}=10.2 \mathrm{~Hz}, \mathrm{~J}_{2}=16.5 \mathrm{~Hz}\right), 3.23\left(\mathrm{~s}, 3 \mathrm{H},-\mathrm{OCH}_{3}\right), 3.67\left(\mathrm{~s}, 3 \mathrm{H},-\mathrm{OCH}_{3}\right), 4.07(\mathrm{q}$, $\left.2 \mathrm{H},-\mathrm{OCH}_{2}-, J=7.2 \mathrm{~Hz}\right), 4.81-4.87(\mathrm{~m}, 2 \mathrm{H}), 6.73-6.77\left(\mathrm{~m}, 2 \mathrm{H}\right.$, aromatics), $6.80\left(\mathrm{~d}, 1 \mathrm{H}_{16}\right.$, aromatic, $\left.\mathrm{J}=4.2 \mathrm{~Hz}\right), 6.92-6.97(\mathrm{~m}$, $2 \mathrm{H}$, aromatics), $7.30(\mathrm{~d}, 1 \mathrm{H}, \mathrm{J}=1.5 \mathrm{~Hz}), 7.69-7.73\left(\mathrm{~m}, 2 \mathrm{H}\right.$, aromatics), $7.82-7.87\left(\mathrm{~m}, 2 \mathrm{H}\right.$, aromatique); ${ }^{13} \mathrm{C} \mathrm{NMR}(75.5 \mathrm{MHz}$, $\left.\mathrm{CDCl}_{3}\right): \delta=14.6\left(\mathrm{CH}_{3}\right), 19.3\left(\mathrm{CH}_{3}\right), 20.7\left(\mathrm{CH}_{3}\right), 28.4(\mathrm{CH}), 39.5\left(\mathrm{CH}_{2}\right), 40.8\left(\mathrm{CH}_{2}\right), 55.7\left(\mathrm{OCH}_{3}\right), 55.9\left(\mathrm{OCH}_{3}\right), 57.5(\mathrm{OOC}-\mathrm{CH}-$ $\mathrm{N}), 64.3\left(\mathrm{OCH}_{2}\right), 81.5(\mathrm{CH}-\mathrm{O}), 108.95\left(\mathrm{CH}_{\text {aromatic }}\right), 111.8\left(\mathrm{CH}_{\text {aromatic }}\right), 113.5\left(\mathrm{CH}_{\text {aromatic }}\right), 120.2\left(\mathrm{C}_{\text {aromatic }}\right), 121.2\left(\mathrm{CH}_{\text {aromatic }}\right)$, 121.3( $\left.\mathrm{C}_{\text {aromatic }}\right), 122.7\left(\mathrm{CH}_{\text {aromatic }}\right), 123.5\left(\mathrm{CH}_{\text {aromatic }}\right), 131.7\left(\mathrm{CH}_{\text {aromatic }}\right), 134.2\left(\mathrm{CH}_{\text {aromatic }}\right), 136.2$ (C 149.3(Caromatic), 150.1 (C aromatic), $150.9\left(\mathrm{C}_{\text {aromatic }}\right.$ ), 156.2(C=N), 166.9(N-C=O), 167.6(COO). TOFMS ES ${ }^{+}$for $\mathrm{C}_{33} \mathrm{H}_{34} \mathrm{~N}_{2} \mathrm{O}_{8}$ theoretical $[\mathrm{M}+\mathrm{H}]^{+}: 587.2315$; measured $[\mathrm{M}+\mathrm{H}]^{+}: 587.2314$.

4-\{[3-(4-ethoxy-3-methoxyphenyl)-4,5-dihydroisoxazol-5-yl]methyl\}-2-methoxyphenyl 2-(1,3-dioxo-1,3-dihydro-2H isoindol-2-yl)-4-methylpentanoate (7cf)

Yellow liquid (62\%). ${ }^{1} \boldsymbol{H}$ NMR $\left(300 \mathrm{MHz}, \mathrm{CDCl}_{3}\right): \delta=0.97\left(\mathrm{~d}, 3 \mathrm{H},-\mathrm{CH}_{3}, \mathrm{~J}=6.6 \mathrm{~Hz}\right), 1.00\left(\mathrm{~d}, 3 \mathrm{H},-\mathrm{CH}_{3}, \mathrm{~J}=6.3 \mathrm{~Hz}\right), 1.45(\mathrm{t}, 3 \mathrm{H}$, $\left.-\mathrm{CH}_{3}, J=7.2 \mathrm{~Hz}\right), 1.58(\mathrm{~m}, 1 \mathrm{H}), 2.03-2.47(\mathrm{~m}, 2 \mathrm{H}), 2.78-2.85\left(\mathrm{dd}, 1 \mathrm{H}, J_{1}=6.9 \mathrm{~Hz}, J_{2}=14.1 \mathrm{~Hz}\right), 2.93-3.01\left(\mathrm{dd}, 1 \mathrm{H}, J_{1}=7.8 \mathrm{~Hz}\right.$, $J_{2}=16.5 \mathrm{~Hz}$ ), $3.07-3.14\left(\mathrm{dd}, 1 \mathrm{H}, J_{1}=6.3 \mathrm{~Hz}, J_{2}=13.8 \mathrm{~Hz}\right), 3.23-3.28\left(\mathrm{dd}, 1 \mathrm{H}, J_{1}=8.1 \mathrm{~Hz}, J_{2}=16.5 \mathrm{~Hz}\right), 3.77\left(\mathrm{~s}, 3 \mathrm{H},-\mathrm{OCH}_{3}\right)$, $3.87\left(\mathrm{~s}, 3 \mathrm{H},-\mathrm{OCH}_{3}\right), 4.10\left(\mathrm{q}, 2 \mathrm{H},-\mathrm{OCH}_{2}, J=6.9 \mathrm{~Hz}\right), 4.88-4.94(\mathrm{~m}, 1 \mathrm{H}), 5.20-5.25\left(\mathrm{dd}, 1 \mathrm{H}, J_{1}=4.5 \mathrm{~Hz}, J_{2}=11.1 \mathrm{~Hz}\right), 6.76-$ $6.99\left(\mathrm{~m}, 5 \mathrm{H}\right.$, aromatics), $7.33(\mathrm{~d}, 1 \mathrm{H}, \mathrm{J}=1.8 \mathrm{~Hz}), 7.71-7.75(\mathrm{~m}, 2 \mathrm{H}$, aromatics $), 7.85-7.88(\mathrm{~m}, 2 \mathrm{H}$, aromatics $) ;{ }^{13} \boldsymbol{C} \boldsymbol{N M R}$ $\left(75.5 \mathrm{MHz}, \mathrm{CDCl}_{3}\right): \delta=14.6\left(\mathrm{CH}_{3}\right), 21.1\left(\mathrm{CH}_{3}\right), 25.1\left(\mathrm{CH}_{3}\right), 26.9(\mathrm{CH}), 39.5\left(\mathrm{CH}_{2}\right), 40.6(\mathrm{CH}), 40.8\left(\mathrm{CH}_{2}\right), 50.6(\mathrm{OOC}-\mathrm{C}-\mathrm{N})$, $55.9\left(2 \mathrm{OCH}_{3}\right), \quad 64.3\left(\mathrm{O}-\mathrm{CH}_{2}\right), \quad 81.5(\mathrm{CH}-\mathrm{O}), \quad 108.9\left(\mathrm{CH}_{\text {aromatic }}\right), \quad 111.7\left(\mathrm{CH}_{\text {aromatic }}\right), \quad 113.6\left(\mathrm{CH}_{\text {aromatic }}\right), \quad 120.2\left(\mathrm{CH}_{\text {aromatic }}\right)$, 121.2( $\left.\mathrm{CH}_{\text {aromatic }}\right), 121.3\left(\mathrm{CH}_{\text {aromatic }}\right), 122.2\left(\mathrm{C}_{\text {aromatic }}\right), 122.5\left(\mathrm{C}_{\text {aromatic }}\right), 123.5\left(\mathrm{CH}_{\text {aromatic }}\right), 129.0$ (C $\left.\mathrm{C}_{\text {aromatic }}\right), 131.8\left(\mathrm{CH}_{\text {aromatic }}\right)$, 134.1 ( $C_{\text {aromatic }}$, 136.2( $C_{\text {aromatic }}$, 138.4( $\mathrm{C}_{\text {aromatic }}$ ), 149.3( $\left.\mathrm{C}_{\text {aromatic }}\right)$ 150.1 ( $\mathrm{C}_{\text {aromatic }}$ ), 156.2( $\left.\mathrm{C}=\mathrm{N}\right), 167.6(\mathrm{~N}-\mathrm{C}=\mathrm{O}), 168.12(\mathrm{COO})$. TOFMS ES ${ }^{+}$for $\mathrm{C}_{34} \mathrm{H}_{36} \mathrm{~N}_{2} \mathrm{O}_{8}$ theoretical $[\mathrm{M}+\mathrm{H}]^{+}: 601.2471 ;$ measured $[\mathrm{M}+\mathrm{H}]^{+}: 601.2467$.

\section{Conclusion}

We have studied the reactivity of allyl esters $5(a-c)$ toward acyclic benzohydroxyaminoyl chlorides $\mathbf{6}(\mathrm{d}-\mathrm{f})$. All cycloadducts were formed in appreciable regiospecificity and chemospecificity, giving the products in good yields. The continuous pharmaceutical interest in the isoxazoline compounds may justify further exploration of these results in the pharmacological field.

\section{ACKNOWLEDGMENTS}

The authors are grateful to the DGRSRT (Direction Générale de la Recherche Scientifique et de la Rénovation Technologique) of the Tunisian Ministry of Higher Education and Scientific Research. 


\section{REFERENCES}

[1] Gaonkar S. L., Rai L. K. M., Prabhuswamy B., Synthesis of novel 3-[5-ethyl-2-(2-phenoxy-ethyl)- pyridin]-5-substituted isoxazoline libraries via 1,3-dipolar cycloaddition and evaluation of antimicrobial activities, Med. Chem. Res. 15 (2007) 407.

[2] Basappa M., Sadashiva P., Mantelingu K., Nanjunda S. S., Rangappa K. S., Solution-phase synthesis of novel $\Delta 2-$ isoxazoline libraries via 1,3-dipolar cycloaddition and their antifungal properties, Bioorg. Med. Chem. 11 (2003) 4539.

[3] Ahmad G., Mishra P. K., Gupta P., Yadav P. P., Tiwari P., Tamrakar A. K., Srivastava A. K., Maurya R., Synthesis of novel benzofuran isoxazolines as protein tyrosine phosphatase 1B inhibitors, Bioorg. Med. Chem. Lett. 16 (2006) 2139.

[4] Conti P., Dallanoce C., Amici M. D., Micheli C. D., Klotz K. N., synthesis of new $\Delta 2$-isoxazoline derivatives and their pharmacological characterization as $\beta$-adrenergic receptor antagonists, Bioorg. Med. Chem. 6 (1998) 401.

[5] Kai H., Matsumoto H., Hattori N., Takase A., Fujiwara T., Sugimoto H., Anti-influenza Virus Activities of 2-AlkoxyiminoN-(2-isoxazolin-3-ylmethyl)acetamides, Bioorg. Med. Chem. Lett. 11 (2001) 1997.

[6] Srivastava S., Bajpai L. K., Batra S., Bhaduri A. P., Maikhuri J. P., Gupta G., In search of new chemical entities with spermicidal and anti-HIV activities, Bioorg. Med. Chem. 7 (1999) 2607.

[7] Ichiba T., Scheuer P. J., Borges K. M., Three bromotyrosine derivatives, one terminating in an unprecedented diketocyclopentenylidene enamine, J. Org. Chem. 58 (1993) 4149.

[8] Habeeb A. G., Rao P. N., Knaus E. E., Design and Synthesis of 4,5-Diphenyl-4-isoxazolines: Novel Inhibitors of Cyclooxygenase-2 with Analgesic and Antiinflammatory Activity, J. Med. Chem. 44 (2001) 2921.

[9] Antczak C., Bauvois B., Monneret C., Florent, A new acivicin prodrug designed for tumor-Targeted delivery, Bioorg. Med. Chem. 9 (2001) 2843.

[10] Maurya R., Ahmad A., Gupta P., Chand k., Kumar M., Preceti R. J., Rasheed N., Palit G., Synthesis of novel isoxazolines via 1,3-dipolar cycloaddition and evaluation of anti-stress activity, Med. Chem. Res. 20(2011) 139.

[11] Simoni D., Grisolia G., Giarmini G., Roberti M., Rondanin R., Piccagli L., Baruchello R., Rossi M., Romagnoli R., Invidiata F. P., Grimaudo S., Jung M. K., Hamel E., Gebbia N., Crosta L., Abbadessa V., Cristina A. D., Dusonchet L., Meli M., Tolomeo M., Heterocyclic and Phenyl Double-Bond-Locked Combretastatin Analogues Possessing Potent ApoptosisInducing Activity in HL60 and in MDR Cell Lines, J. Med. Chem. 48 (2005) 723-736.

[12] Huisgen R., 1,3-Dipolar Cycloadditions. Past and Future, Angew. Chem., Int. Ed. Engl., 2 (1963) 565-598.

[13] Frederickson M., Optically active isoxazolidines via asymmetric cycloaddition reactions of nitrones with alkenes: applications in organic synthesis, Tetrahedron, 53 (1997) 403-425.

[14] For a general review of this area see: (a) Tufariello J. J., 1,3-Dipolar Cycloaddition Chemistry In Padwa, A., Ed.; Wiley: New York, 1984; (b) Torssell K. B. G., Nitrile Oxides, Nitrones and Nitronates in Organic Synthesis; VCH: New York, 1988.

[15] (a) Seerden J. P. G., Boeren M. M. M., Scheeren H. W., 1,3-Dipolar cycloaddition reactions of nitrones with alkyl vinyl ethers catalyzed by chiral oxazaborolidines, Tetrahedron, 53(1997) 11843-11852; (b) Gothelf K. V., Jørgensen K. A., Asymmetric 1,3-Dipolar Cycloaddition Reactions, Chem. Rev., , 98 (1998) 863-910.

[16] Wagner H., Jurcic K., Deininger R., Planta Med., 1979, 37, 9-14.

[17] Feng J., Lipton J. M., Eugenol: Antipyretic activity in rabbits, Neuropharmacology, 26 (1987) 1775-1778.

[18] Hume W. R., Effect of Eugenol on Constrictor Responses in Blood Vessels of the Rabbit Ear, J. Dent. Res. , 62 (1983) 1013-1015.

[19] Shattuck J., Shreve C., Solomon S., Enantioselective Synthesis of Imperanene, a Platelet Aggregation Inhibitor ,Org Lett. 3 (2001) 3021.

[20] Essersi A., Touati R., Ben Hassine B., Diastereoselective Synthesis of $\gamma$-Phthalimido- $\beta$-Hydroxy Esters and NProtected 4-Amino-1,3-Diols Starting from Natural a-Amino Acids, Letters in Organic Chemistry, 7 (2010) 69.

[21] Liu K. C., Shelton R. B., Howe R. K., A particularly convenient preparation of benzohydroximinoyl chlorides (nitrile oxide precursors), J. Org. Chem. 45 (1980) 3916; Huisgen R., Mack N., 1.3 Dipolare additionen der nitriloxyde an carbonylverbindungen, Tetrahedron Lett. 2 (1961) 583.

[22] Lorand K., Melinda N., Enik F., Reijo S., Ferenc F., Synthesis of novel isoxazoline-fused cispentacin stereoisomers, Tetrahedron Lett. 50 (2009) 2605-2608.

[23] Juneau S., Synthèse asymétrique de 2-isoxazolines en vue de la synthèse totale de la négamycine, Université du Québec à Montréal, 2008.

[24] Lahouar G., Touati R., Khamri S., Marrot J., Ben Hassine B., Asymmetric synthesis and spectroscopic characterization of various $\mathrm{N}$-substituted phthaloyl and tosyl derivatives, Journal of Chemical and Pharmaceutical Research,7 (2015) 283-288.

[25] Jegham N., Kacem Y., Ben Hassine B., Regioselective Synthesis and Structure of New Spiro-isoquinolinedione Derivatives, Heterocycles, 81 (2010) 707-715.

\section{Figures captions}

Figure 1- Representative examples of anti-cancer isoxazoline derivatives.

Scheme 1- Synthesis of the optically pure esters derived from eugenol and amino acid.

Scheme 2-1,3-Dipolar cycloaddition reaction of benzohydroxyaminoyl chlorides 6(d-f) with allyl esters 5(a-c).

Scheme 3- Regiochemistry of cycloaddition. 